\title{
Fahd Al-Mulla (ed). Formalin-Fixed Paraffin-Embedded Tissue. Methods and Protocols. Methods in Molecular Biology 724. The Humana Press, ISBN 978-1-61779-054-6
}

\author{
Flavia Troglio $\cdot$ Manuela Vecchi
}

Published online: 3 February 2012

(C) Springer Science+Business Media, LLC 2012

This book is not only a detailed technical manual for a variety of different molecular analyses that can be applied to formalin-fixed paraffin-embedded (FFPE) tissues, but is also a brief guide to the critical regulatory and ethical implications associated with the use of human archival fixed samples for research purposes. Indeed, the first chapter illustrates how FFPE archival tissues and their related clinical and diagnostic records represent an invaluable source of information. At the same time, it describes the lively debate regarding the need, in our era of personalized medicine, for a unified regulatory or bioethical policy establishing desirable national and international standards that cover the spectrum of research activities and associated results with FFPE tissue specimens.

The remaining sections illustrate recently developed techniques for expression studies on FFPE tissues comprising tissue microarray construction, particularly appropriate for high-throughput procedures, microarray-based CGH and copy number analysis, gene expression profiling, mutational analysis, methylation profiling and protein mass spectrometry as well as cytological methodologies including multiple immunofluorescence labeling and in situ hybridization.

The book also provides a detailed description of currently available protocols for the extraction of biological material that is generally degraded in FFPE samples, including laser-capture microdissected tissues and presents a number of alternative tissue fixatives to formalin fixation to cater for current molecular applications.

One chapter also addresses the critical aspects of reproducibility and standardization of immunohistology between different laboratories. This concept is generally underestimated, but of extreme importance, given the many variables that impact on antigen preservation in paraffinembedded biopsies.

While being very detailed in the practical procedures, which include troubleshooting sections and accurate reagent descriptions, not all chapters provide a critical discussion on the quality of the data produced using different techniques, nor do they present a thorough comparative analysis of different protocols and methodologies.

This text can be considered an introductory compendium of current methods and protocols in Molecular Biology for the use of fixed paraffin-embedded tissues that will be particularly suited to new researchers in the field who are eager to develop expertise. It is an easily readable and technically comprehensive book that would be ideal for initial experimental planning which can be complemented by other bibliographic sources and scientific journals for in-depth discussions of particular techniques and their potential applications.
F. Troglio $(\bowtie) \cdot$ M. Vecchi

Molecular Medicine Program, European Institute of Oncology,

Via Ripamonti 435, 20100 Milan, Italy

e-mail: flavia.troglio@ifom-ieo-campus.it

M. Vecchi

e-mail: manuela.vecchi@ifom-ieo-campus.it 